\title{
Effect of Sb Addition on the Mechanical and Structural Behaviour of Sn-Bi alloy
}

\author{
F.Abd El-Salam, M.M. Mostafa, R.H. Nada, H.S.Mohammed
}

Faculty of Education, Physics Dept., Ain Shams Univ., Cairo, Roxy.

Sn-12wt.\% Bi (alloy A) and Sn-12wt.\% Bi-1wt.\%Sb (alloy B) solder alloys homogenized at $463 \mathrm{~K}$ for $24 \mathrm{~h}$ were annealed for $2 \mathrm{~h}$ at $443 \mathrm{~K}$. From both alloys a group of samples was slowly cooled to room temperature with a cooling rate of $1.2 \times 10^{-2} \mathrm{~K} / \mathrm{s}$. A second group of samples was rapidly quenched in cold water (273K). Stress-strain, $\sigma-\varepsilon$, tests performed in the temperature range from (333-413) $\mathrm{K}$ in steps of $10 \mathrm{~K}$, showed a temperature dependence of the hardening behaviour with minimum at $353 \mathrm{~K}$ followed by a peak at $363 \mathrm{~K}$ above which showed continuous decrease. The softening behaviour increased to $353 \mathrm{~K}$ then showed minimum at $363 \mathrm{~K}$ followed by continuous increase at higher temperatures for both alloys. In the low temperature range the softening coefficient, $\alpha$, increased with Sb addition and its values were higher for the slowly cooled samples than the quenched samples. The intrinsic hardness at $0 K, H_{o}$, was higher for the tertiary and quenched samples all over the tested temperature range. In the high temperature range, $\alpha$ showed a constant value of .02, Ho showed different values at $0 K$ due to the different initial states of the tested samples. Increasing the temperature and Sb addition lead to more softening. The activation energy for the annealed and quenched samples of both alloys were found to be 16.72 and $19.144 \mathrm{~kJ} / \mathrm{mol}$ for the low and high temperature regions, respectively. The average lattice parameters, " $a$ ", $\frac{\Delta a}{a}$, of the Sn-rich phase showed two peaks at $(343,383) K$ and minima at $363 k$, while "c", $\frac{\Delta c}{c}$ and the ratio cla decreased to minima at $353 \mathrm{k}$ and exhibited peaks at $(343,363) k$ then decreased with further temperature increase.

\section{Introduction:}

The most commonly soldering materials used in electronic interconnection and packing are the $\mathrm{Pb}$-Sn systems because of their low cost and unique combination of physical, chemical, mechanical properties, manufacturability and reliability. Because of environmental and health concerns, due to the toxity of $\mathrm{Pb}$ and its compound [1], the intensive search for alternative $\mathrm{Pb}$-free solder alloys is in progress. 
The low hardness and consequently the low mechanical strength of pure Sn make it suitable for use as a bulk material for construction unless strengthened by hardening alloy elements [2]. This is carried out by replacing the toxic $\mathrm{Pb}$ with non-toxic elements like $\mathrm{Bi}, \mathrm{Ag}$, and $\mathrm{Cu}$ to get intended properties suitable in different applications [3].

The most of the candidate $\mathrm{Pb}$-free solders are $\mathrm{Sn}$-rich, typically containing more than $90 \% \mathrm{Sn}$, which are known as, pewters. This suggests that the physical, chemical, and mechanical properties of the proposed $\mathrm{Pb}$-free solders are heavily influenced by the properties of pure $\mathrm{Sn}$, in contrast to $\mathrm{Sn}-\mathrm{Pb}$ eutectic, which comprises a mixture of Sn-rich and $\mathrm{Pb}$-rich phases [4].

$\mathrm{Sn}-\mathrm{Bi}$ alloys, which outperform the $\mathrm{Sn}-\mathrm{Pb}$ alloys, attracted the attention because of their good properties as solders for some delicate electronic tools [5].

It was reported [6] that $\mathrm{Bi}$ can be added to $\mathrm{Sn}$ as a major element and Sn-57wt\% Bi binary eutectic solder alloy is known as a useful low-melting temperature solder (melting at $139 \mathrm{C}^{\circ}$ ), which is lower than melting temperature of eutectic $\mathrm{Pb}-\mathrm{Sn}$; and by adding a small a mounts of third or fourth alloying elements to the solder is one of the best ways to improve mechanical properties.

The Bi-Sb-Sn system as $\mathrm{Pb}$-free solder candidate is considered as basic system for development of a new $\mathrm{Pb}$-free soldering materials on the basis of the thermodynamics database developed [7].

Creep tests of $\mathrm{Sn}-5 \mathrm{wt} \% \mathrm{Bi}$ revealed a decomposition of the alloy at $328 \mathrm{~K}$ into a solid solution and pure $\mathrm{Bi}$. Both the strain rate sensitivity parameter changes and the values of the activation energy obtained before and after precipitation of the $\mathrm{Bi}$ phase were attributed to a grain boundary sliding mechanism [8]. A decrease in the steady state creep rate and an increase in the activation volume were obtained [9] with increasing grain size for $\mathrm{Sn}-0.5$ at. \% Bi alloy. This was interpreted as being due to the segregation of the solute atoms (Bi) in the vicinity of the grain boundaries as well as on the moving dislocations.

By performing creep tests for $\mathrm{Sn}-3 \mathrm{wt} \% \mathrm{Bi}$ alloy it is expected that in the low temperature region (below $330 \mathrm{~K}$ ), $\alpha$-phase (Sn-rich phase) and $\beta$-phase (Bi-rich phase) coarsen and the solubility of $\mathrm{Bi}$ in $\mathrm{Sn}$ increase with increasing the testing temperature until the transition temperature $(330 \mathrm{~K})$ after which the $\beta$-phase dissolves completely and disappears [10]. The gradual increase in both the transient creep parameters $\beta$ and $n$ in the low temperature range (300-330 $\mathrm{K})$ may be attributed to the redistribution and coarsening of Bi-rich particles. The rapid increase of these parameters in the high temperature range (330-370 
$\mathrm{K})$ may be due to the dissolution of Bi-rich particles and the homogenization in the alloy.

The results of creep deformation of Sn-5wt\%Bi alloy samples in the temperature range $308-388 \mathrm{k}$ revealed three structures existing in three temperature ranges. In the first range, $308-328 \mathrm{~K}, \alpha$-phase (rich in Bi) exists, in the range, $328-361 \mathrm{~K},(\alpha+\beta)$ phases exist. Above $361 \mathrm{~K}$ up to $388 \mathrm{~K}$, only the $\beta$ phase (Sn- rich) exists. These stages were due to two transition temperatures at $328 \mathrm{~K}$ and $361 \mathrm{~K}$ characterized by the increased values of the strain sensitivity parameters above these temperatures, as supported by the observed peaks in the measured X-ray diffraction parameters at these temperatures. The data showed that the behaviour of the temperature dependence of grain diameter, GD, was opposite to that of the parameter, a, all over the tested temperature range, while that of the parameter, c, was opposite to that of, GD, up to $348 \mathrm{~K}$ above which it was matched with it up to $388 \mathrm{~K}$. It was also found that the lattice strain due to the parameter $\left(a=\frac{\Delta a}{a_{o}}\right)$ is large compared to that due to $\left(c=\frac{\Delta c}{c_{o}}\right)$ in the same temperature range [11].

The addition of $1 \mathrm{wt} \% \mathrm{Ag}$ to $\mathrm{Sn}-7 \mathrm{wt} \% \mathrm{Bi}$ [5], significantly increased in the ductility and softening was observed in the Ag doped alloy compared to the $\mathrm{Ag}$ free binary alloy. The addition of $1 \mathrm{wt} \% \mathrm{Ag}$ effectively inhibits the uncontrolled growth of the high strength large structure during solidification of binary Sn-Bi alloy.

Creep parameters for $\mathrm{Sn}-3 \mathrm{wt} \%$ Bi alloy samples measured under different applied stresses ranging from 13.24 to $16.30 \mathrm{MPa}$ in the temperature range from $300-370 \mathrm{~K}$, increased with increasing stress and/or temperature . The gradual increase observed below the transition temperature $(330 \mathrm{k})$ was attributed to redistribution and coarsening of $\beta$-rich particles. Above $330 \mathrm{~K}$ the rapid increase was rendered to the dissolution of Bi-rich particles in the $\alpha$-phase formed at high temperatures leading to the softening observed due to a decrease in the free Bi existing in the alloy matrix [12].

The present work aims at studying the stress-strain behaviour of Sn-12wt. $\% \mathrm{Bi}$ alloy at different temperatures and the variations occurring on adding $1 \mathrm{wt}$. $\% \mathrm{Sb}$ under the same conditions for the binary alloy. The structural variations are also investigated by $\mathrm{x}$-ray diffraction analysis. Bi was chosen because of its surface activity with respect to $\mathrm{Sn}$ [13].

\section{Experimental Procedure:}


The investigated materials Sn-12wt $\%$ Bi (alloy A) and Sn- $12 \mathrm{wt} \%$ Bi-1wt\%Sb (alloy B) were prepared from pure elements $99.99 \%$, by melting the constituents in a high purity graphite crucible. The ingots were obtained in the form of rods. After annealing for homogenization at $463 \mathrm{~K}$ for $24 \mathrm{~h}$, the ingots were drawn to wire of $0.7 \mathrm{~mm}$ diameter. Two groups of samples of each alloy were annealed for $2 \mathrm{~h}$ at $443 \mathrm{~K}$ to eliminate the cold work introduced during swaging. Then group A of samples of each alloy was slowly cooled to room temperature with a cooling rate of $1.2 \times 10^{-2} \mathrm{~K} / \mathrm{s}$ to obtain mixed $(\alpha+\beta)$ phases and become fully precipitated samples as clear from the phase diagram of Fig.(1).

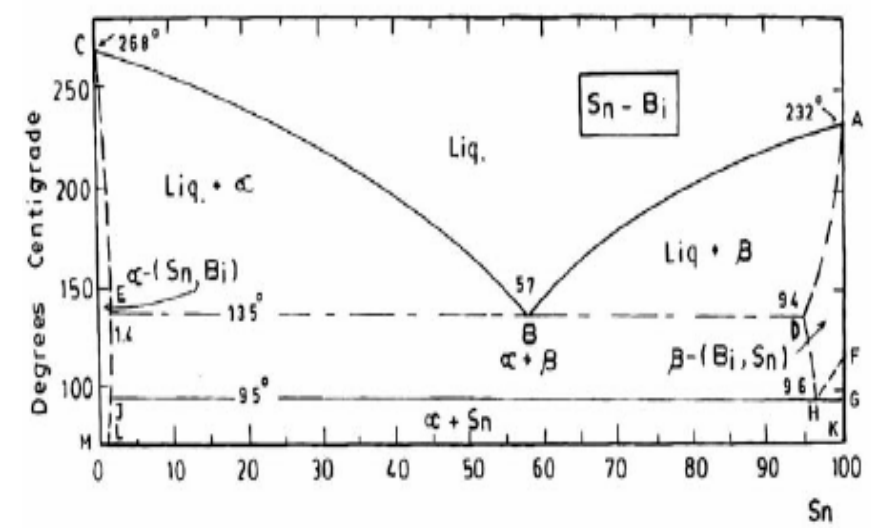

Fig.(1) Sn-Bi phase diagram

The second group of samples of each alloy was rapidly quenched in cold water $(273 \mathrm{~K})$ to obtain samples with supersaturated $\alpha$-solid solution structure [14].

Stress-strain tests were preformed at the temperature range from (333-413) $\mathrm{K}$ in steps of $10 \mathrm{~K}$ using a conventional tensile testing machine described else where [15]. The applied stress was increased gradually with $60 \mathrm{sec}$ interval between two successive loadings and the corresponding elongation was immediately recorded using a dial gauge with accuracy $0.01 \mathrm{~mm}$. A conventional tensile testing machine described else where was used [15]. The applied stress was increased gradually with $60 \mathrm{sec}$ interval between two successive loadings and the corresponding elongation was immediately recorded using a dial gauge with accuracy $0.01 \mathrm{~mm}$, reached.

The fracture stress $\sigma_{\mathrm{f}}$ was found from the maximum stress of the stress-strain curve whose linear part was extrapolated to zero strain to 
determine the yield stress (The macroscopic elastic limit), $\sigma_{y}$ [16]. The slope of the starting linear part of the curve; $\partial \sigma / \partial \varepsilon$; is the Young's modulus, $Y$, and the time of a full stress-strain run till fracture was taken as the fracture time $t_{f}$. The work-hardening coefficient, $\chi$, obtained as the slope of the straight line of the relation between $\sigma^{2}$ and $\varepsilon$ in the parabolic region of the stress-strain curve, proves the applicability of the following equation [17]:

$$
\chi=\frac{\delta \sigma^{2}}{\delta \varepsilon}=G^{2} \frac{b}{2 \Pi^{2} L}
$$

where $\mathrm{G}$ is the shear modulus of the material $(\mathrm{G}=18.3 \mathrm{GPa})$ and, $b$ is the Burgers vector $(b \cong 0.317 \mathrm{~nm})[18]$ and $\mathrm{L}$ is the distance slipped by dislocation.

The resilience, which is the ability of a material to absorb energy when deformed elastically and return it when unloaded, is generally measured by the modulus of resilience $u_{R}$ given as [19a]:

$$
u_{R}=\frac{\sigma_{y} \varepsilon_{y}}{2}=\frac{\left[\sigma_{y} \times\left(\sigma_{y} / Y\right)\right]}{2}=\frac{\sigma_{y}^{2}}{2 Y}
$$

The ability of a material to absorb energy $\mathrm{u}_{\mathrm{T}}$ in the plastic range, which is considered as the total area under the stress-strain curve, is called the toughness of the material. For a parabolic stress-strain curve the area under the curve, or the amount of the work done per unit volume of the material without causing it to rupture is given as [19a]:

$$
u_{T}=\frac{2 \sigma_{u} \varepsilon_{f}}{3}
$$

where $\sigma_{\mathrm{u}}$, or simply the fracture stress $\sigma_{\mathrm{f}}$, and $\varepsilon_{\mathrm{f}}$ is the fracture strain. $\mathrm{u}_{\mathrm{R}}$, and $\mathrm{u}_{\mathrm{T}}$ can be considered as hardening parameters since their values are calculated in terms of the stress, $\sigma$, and the strain, $\varepsilon$.

The increasing demand for materials to withstand higher temperatures, as those used for cladding fuel elements in water cooled reactors makes hardness, $\mathrm{H}$, measurement a handy tool since it is related in several alloy systems with other mechanical properties such as the yield stress $\sigma_{\mathrm{y}}$, which is intimately related to $\mathrm{H}$ and typically, $\mathrm{H}=3 \sigma_{\mathrm{y}}$, in bulk metals [20]. Also, the variation of hardness with temperature was reported as [15]

$$
\mathrm{H}=\mathrm{H}_{\mathrm{o}} \exp (-\alpha \mathrm{T})
$$

It is clear that the temperature dependence behaviour of hardeness, $\mathrm{H}$, is similar to that of yield stress, $\sigma_{y}$.

The microstructure variations of the quenched samples of both alloys A and B were identified after deformation using a Philips X-ray diffractometer 
(PW 3050/60), with $\mathrm{Cu} \mathrm{K}_{\alpha}$ radiation of wave length $0.15425 \mathrm{~A}^{\circ}$ due to nickel filter.

\section{Experimental Results:}

Stress-strain curves obtained at temperatures in the range from $(333-413) \mathrm{K}$ for slowly cooled and quenched $\mathrm{Sn}-12 \mathrm{wt} \% \mathrm{Bi}$ and $\mathrm{Sn}-12 \mathrm{wt} \% \mathrm{Bi}$ $1 \mathrm{wt} \% \mathrm{Sb}$ samples are shown in Fig.(2). It is clear that the levels of the curves are shifted monotonically towards lower flow stress and increased total fracture strain (higher ductility), with increasing deformation temperature. The monotonic shift is interrupted at $363 \mathrm{~K}$ for both alloys. All the curves have parabolic region above the elastic limit.

Induced thermal softening in Fig.(2) for both alloys is revealed from:

i. The decreasing behaviour of the temperature dependence of the hardening parameters: $\sigma_{\mathrm{y}}, \sigma_{\mathrm{f}}, \chi, \mathrm{t}_{\mathrm{f}}, \mathrm{Y}, \mathrm{u}_{\mathrm{R}}$, and $\mathrm{u}_{\mathrm{T}}$, given in Fig.3(a-g) exhibited minima at $353 \mathrm{~K}$, for both alloys.

ii. The increasing softening behaviour of the softening parameters $\varepsilon_{\mathrm{f}}, \dot{\varepsilon_{\mathrm{f}}}$ and $\mathrm{L}$ with increasing deformation temperature, up to $353 \mathrm{~K}$ then show minima at $363 \mathrm{~K}$ followed by continuous increase at higher temperatures as shown in Fig. (4).

In general, the $\mathrm{Sb}$-free alloys are softer than the tertiary alloys.

The temperature dependence of the parabolic work hardening parameter $\chi$ of the tested samples is shown in Fig.(3c). For the quenched tertiary alloy the values of, $\chi$, are lower than those for the slowly cooled tertiary alloy and the quenched binary alloy.

According to the data of $\sigma_{\mathrm{y}}$ given in Fig.(3.a), for the tested alloys the behaviour of the temperature dependence of the hardness, H, given in Fig.(5.a) is similar to that for $\sigma_{y}$ given in Fig.(3.a), i.e., increasing temperature decreases H. Applying Eq. (4), from the relation between $\mathrm{lnH}$ and T given in Fig.(5b). The obtained values of $\mathrm{H}_{\mathrm{o}}$ and $\alpha$ for both alloys are given in Table (1). 


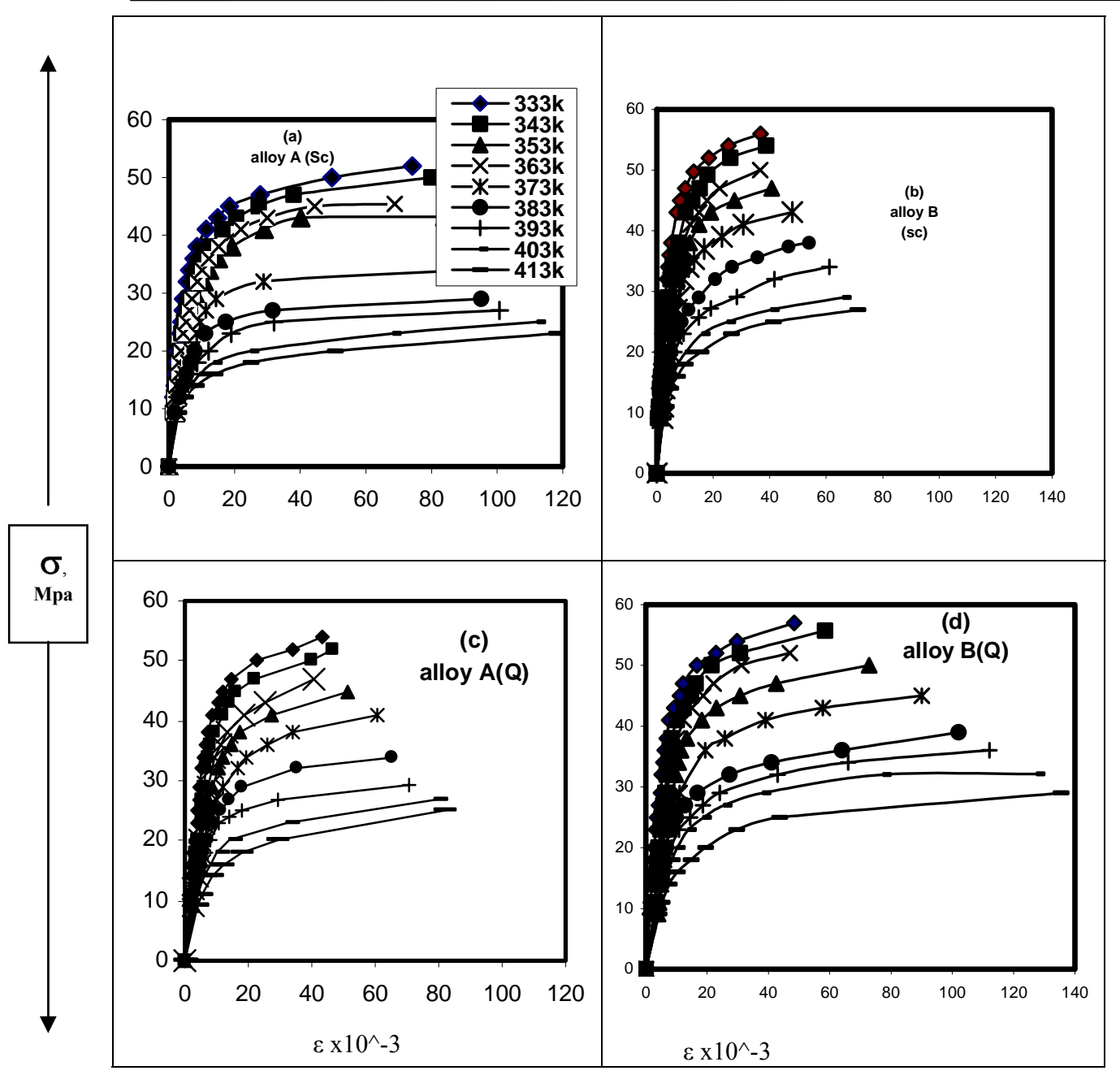

Fig.(2): The stress-strain, $\sigma-E$, curves at different working temperatures for slowly cooled and quenched both alloys. 

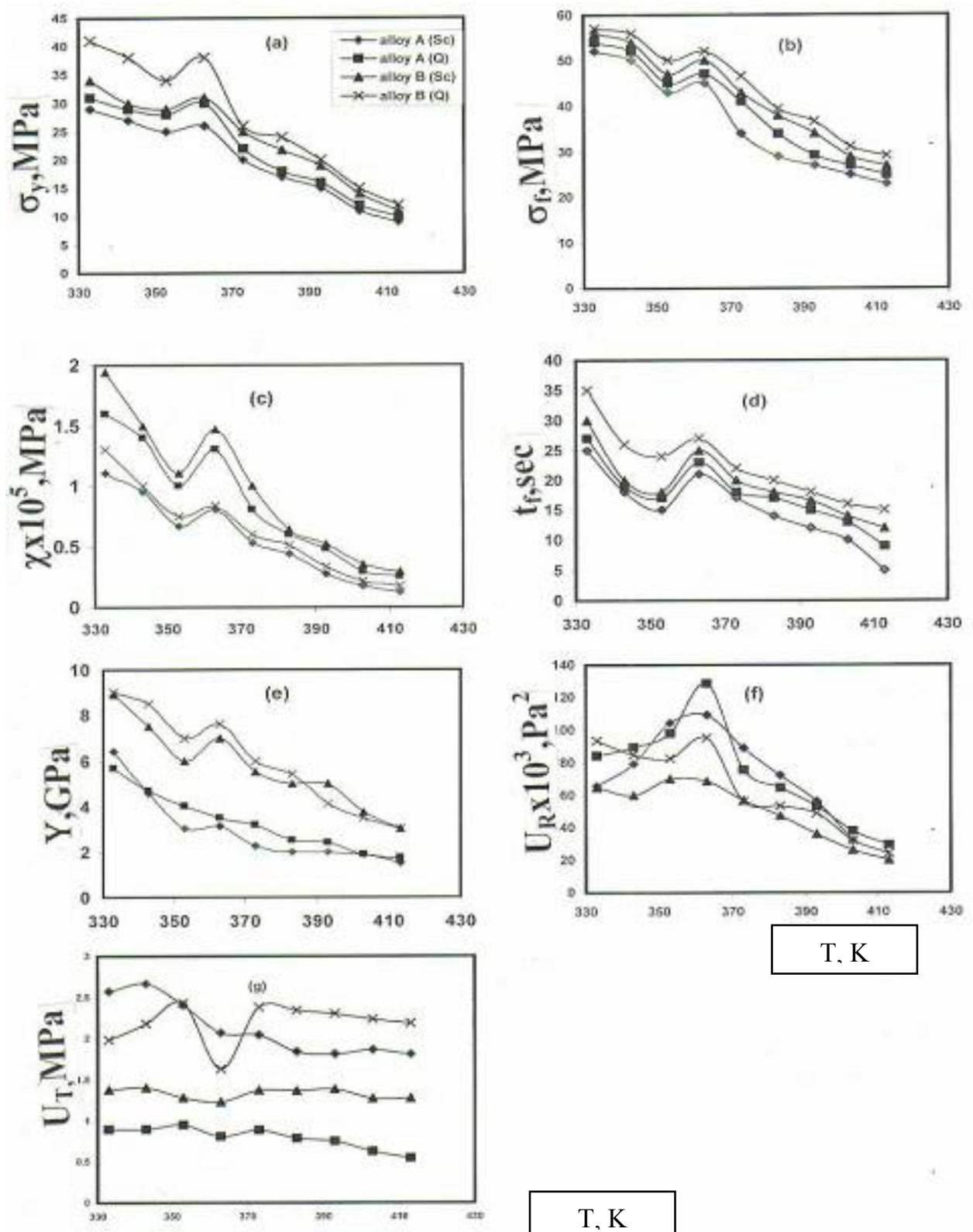

\section{$\mathrm{T}, \mathrm{K}$}

Fig. (3). The temperature dependence of (a) $\sigma_{y},(b) \sigma_{f}$, (c) $\chi$, (d) $t_{f},(e), Y,(f) u_{R},(g) u_{T}$ for alloys A and B. 

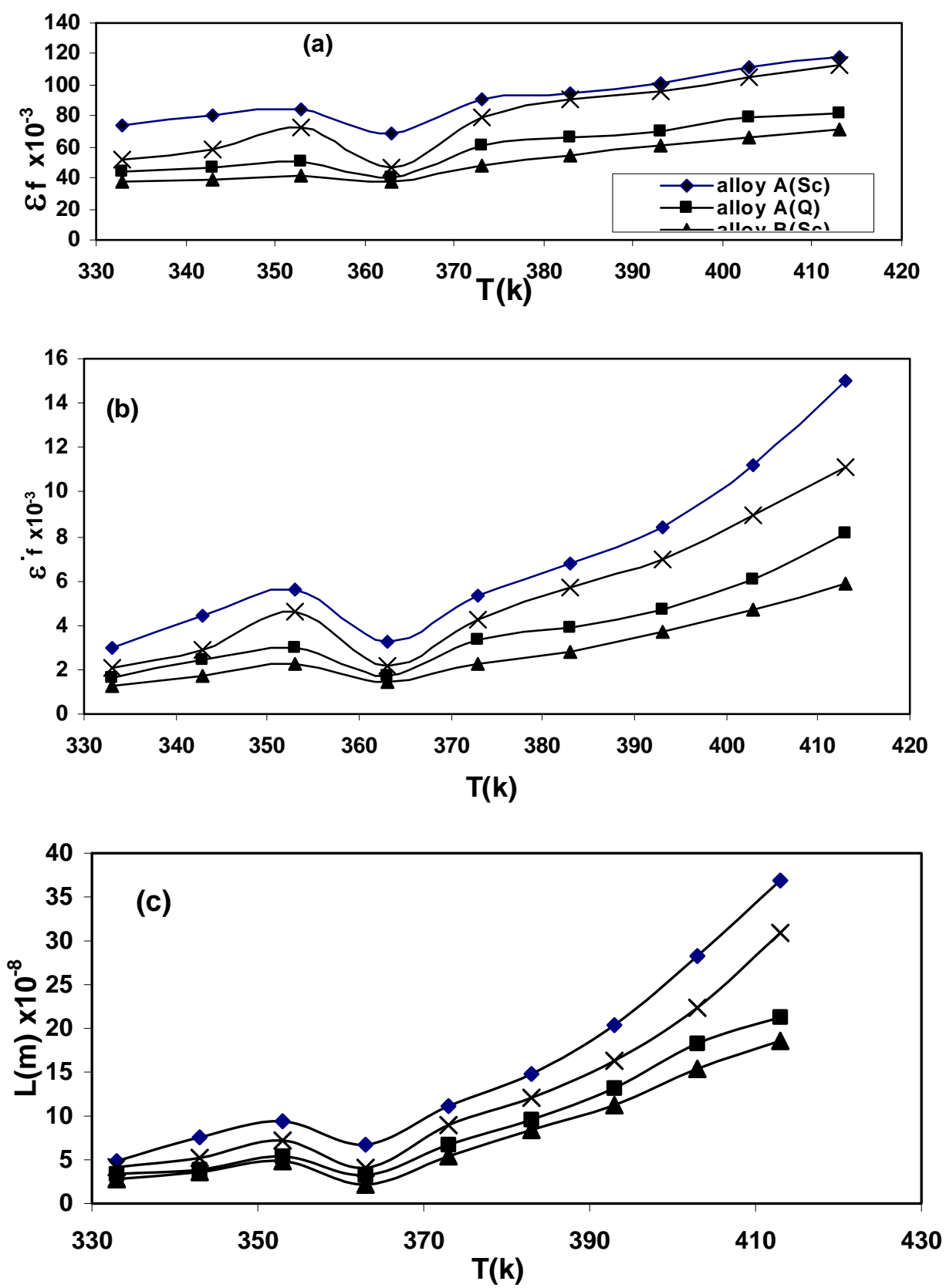

Fig. (4). The temperature dependence of (a) $\varepsilon_{f},(b) \varepsilon_{f}^{\cdot}$, (c) $L$ for alloys $A$ and $B$. 

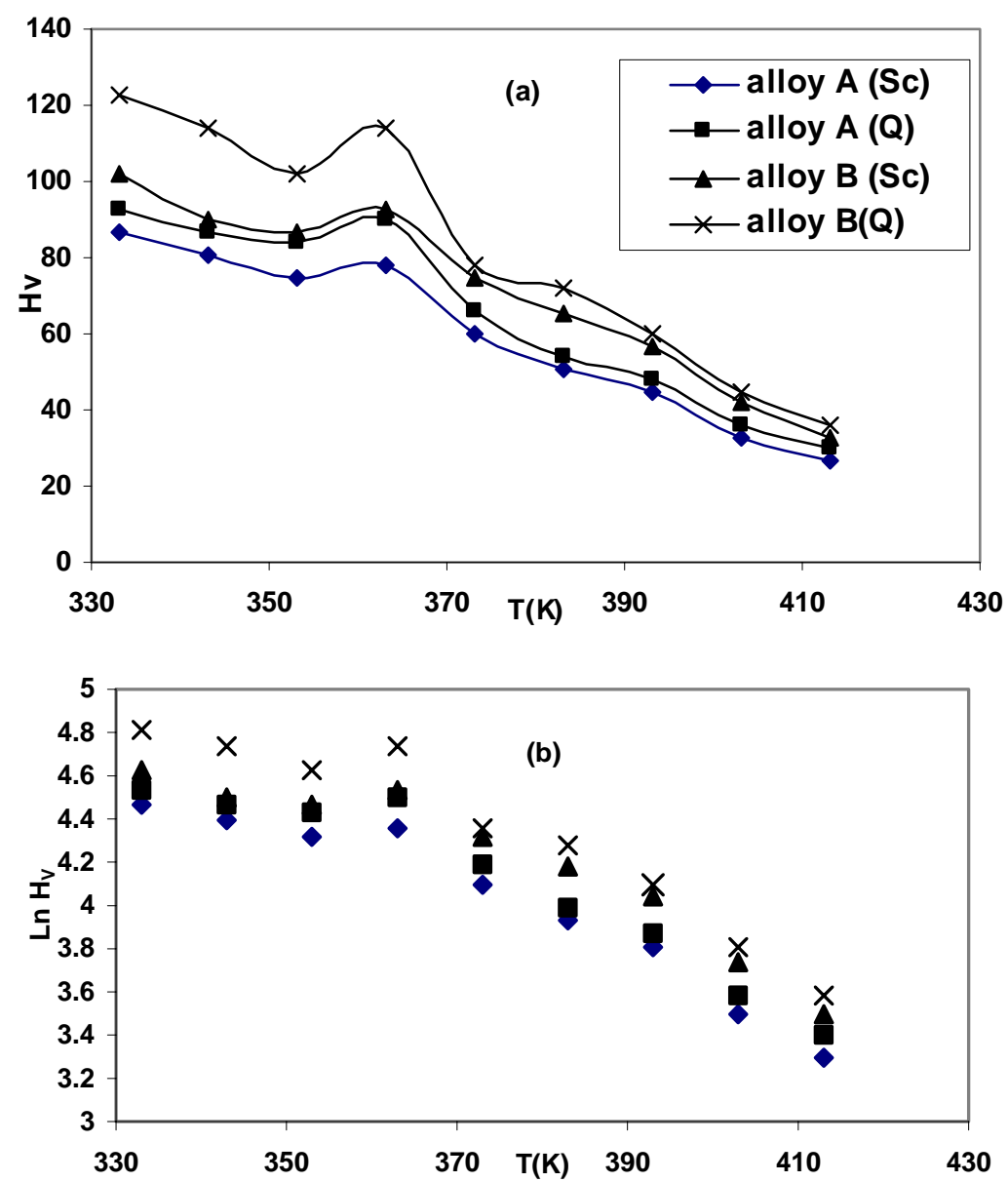

Fig. (5). the temperature dependence of (a) $\mathrm{H}$ and (b) $\mathrm{InH}$ for alloys $\mathrm{A}$ and $\mathrm{B}$.

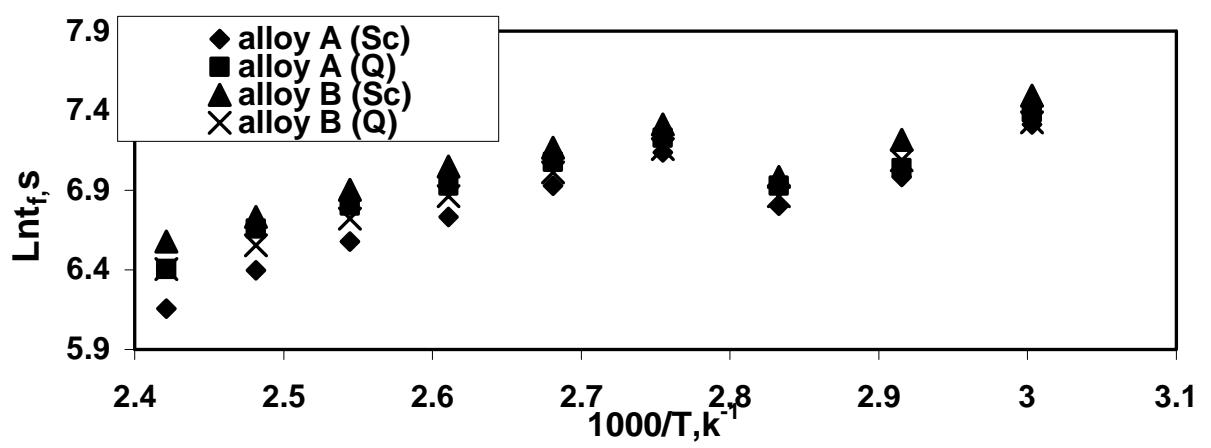

Fig.(6): The relation between $\ln t_{f}$ and 1000/T for alloys A and B. 
The energy activating the fracture mechanism in the present work is calculated assuming that the fracture time $t_{f}$, varies with the working temperature according to the Arrhenius-type relation [17, 21]:

$$
t_{f}=\text { Const } \quad . \exp \left(\frac{Q}{k T}\right)
$$

where $\mathrm{Q}$ is the activation energy $(\mathrm{kJ} / \mathrm{mol})$ and $\mathrm{k}$ is the Boltzmann constant. The plots of $\ln t_{f}$ versus $1000 / T_{a}\left(K^{-1}\right)$ for the alloys A and B given in Fig.(6) show two temperature regions. The values of the activation energy obtained from the slopes of Fig.(6) for the annealed and quenched samples of both alloys were found to be 16.72 and $19.144 \mathrm{~kJ} / \mathrm{mol}$ for the low and high temperature regions, respectively.

The microstructure parameters for the quenched samples of both alloys A and $\mathrm{B}$ were examined by using X-ray technique. The X-ray analysis indicated only the presence of Sn-matrix. The integral X-ray diffraction intensity (I) and the values of the full line-width at half-maximum intensity $(\Delta 2 \theta)$, as calculated from the values of all the reflecting planes for the Sn-rich phase are given in Fig.(7). The values of $(\Delta 2 \theta)$ were found to change with increasing working temperature and exhibit minimum value at $363 \mathrm{k}$. The average lattice parameter, "a" and the residual lattice strain $\frac{\Delta a}{a}$, of the Sn-rich phase shows slight decrease with increasing temperature till a minimum at $363 \mathrm{k}$ then increases with further temperature increase as shown in Fig.8 (a,b). The lattice parameter "c" and the residual lattice strain $\frac{\Delta c}{c}$ and the ratio c/a decrease with increasing working temperature and exhibit peak values at 363k as shown in Fig.9 (a, b).

\section{Discussion:}

According to the Sn-Bi phase diagram, Fig.(1), thermally induced structure variations take place in the temperature range (333-413) K, within which lie the working temperatures of the present work, so the measured parameters prove that they are structure sensitive parameters.

The strength of binary alloy increases with increasing second phase content or adding third alloying element which decrease dislocation mobility.

At certain temperature the thermally induced phases will be in general of the same features whether they stem from a formation or a 
dissolution process. Hence, the controlling factor for strength of an alloy will be the existing amount of the phase and the nature of the surrounding matrix [15].
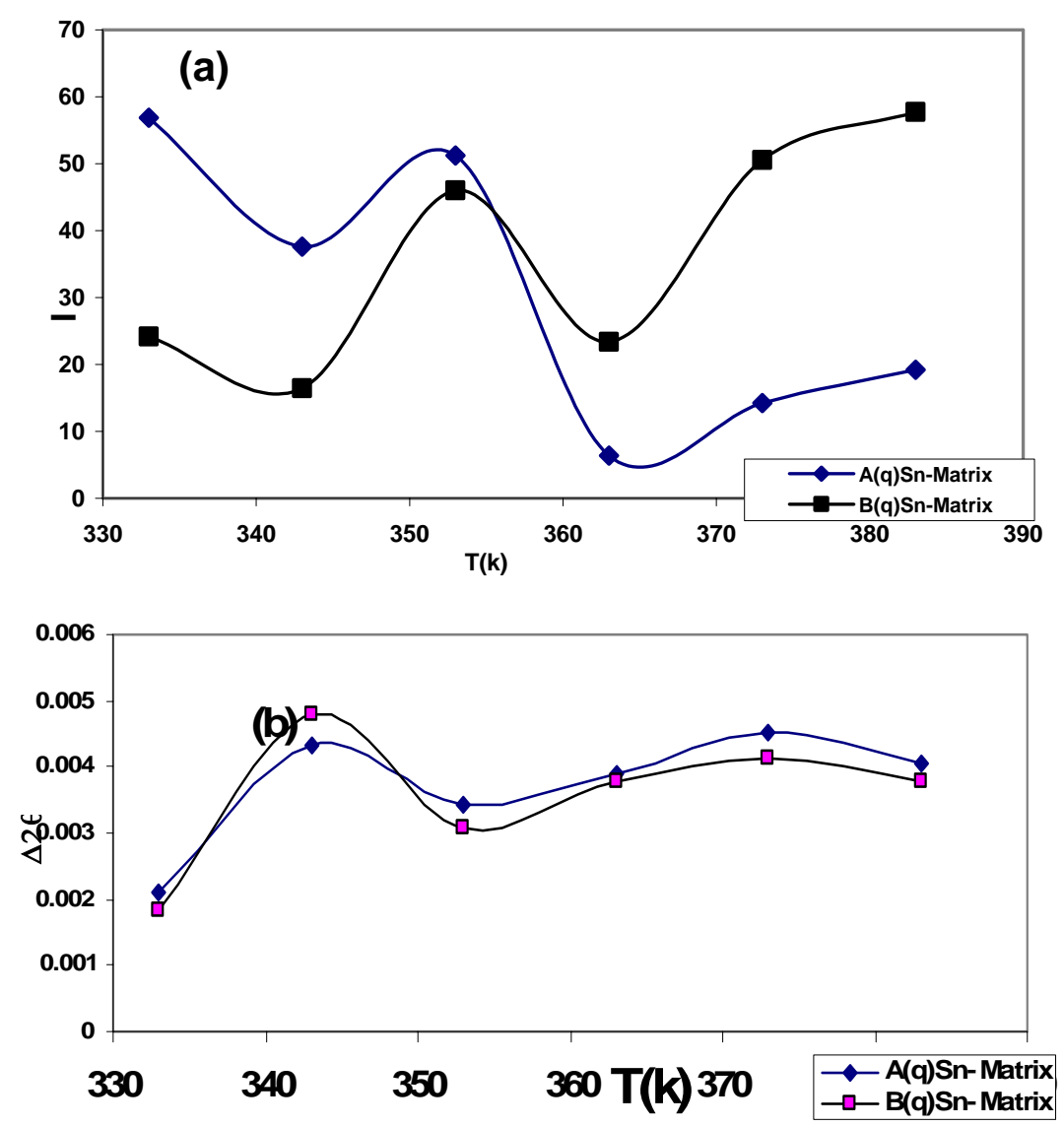

Fig.(7): The effect of $\mathrm{T}(\mathrm{K})$ on intensity (I) and $(\Delta 2 \theta)$ for both alloys. 

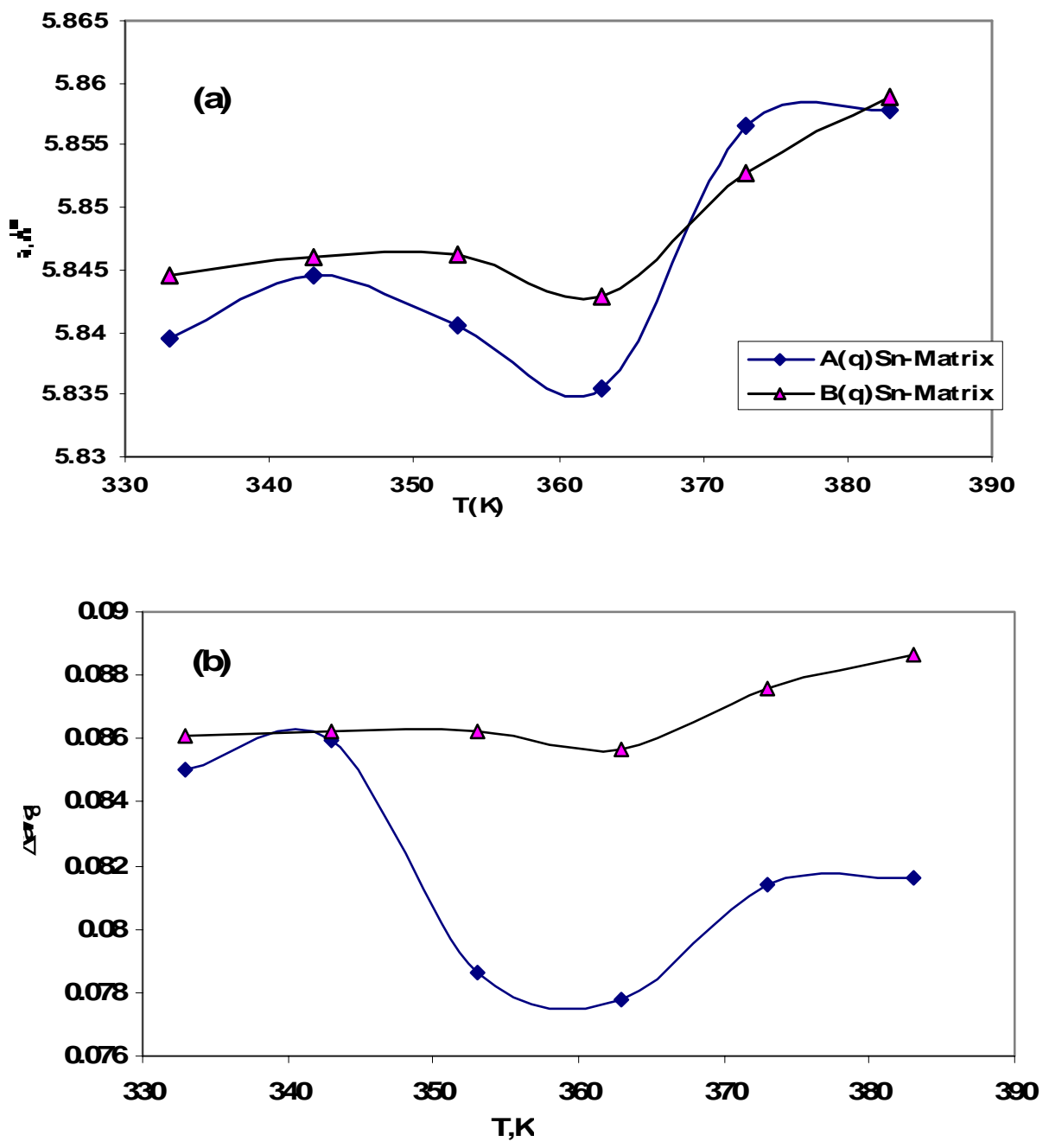

Fig. (8a, b) The effect of temperatures on the lattice parameter "a" and the residual lattice strain $\frac{\Delta a}{a}$ for both alloys.

Intrinsic point defects in thermal equilibrium and concentration determined by Boltzman's factor, (-E/KT), are expected to from during preparation in ratios depending on the heat treatment and cooling regime.

According to the surface activity theory [13], Bi and $\mathrm{Sb}$ atoms concentrate at the cores of grains leaving more mobile boundaries. 

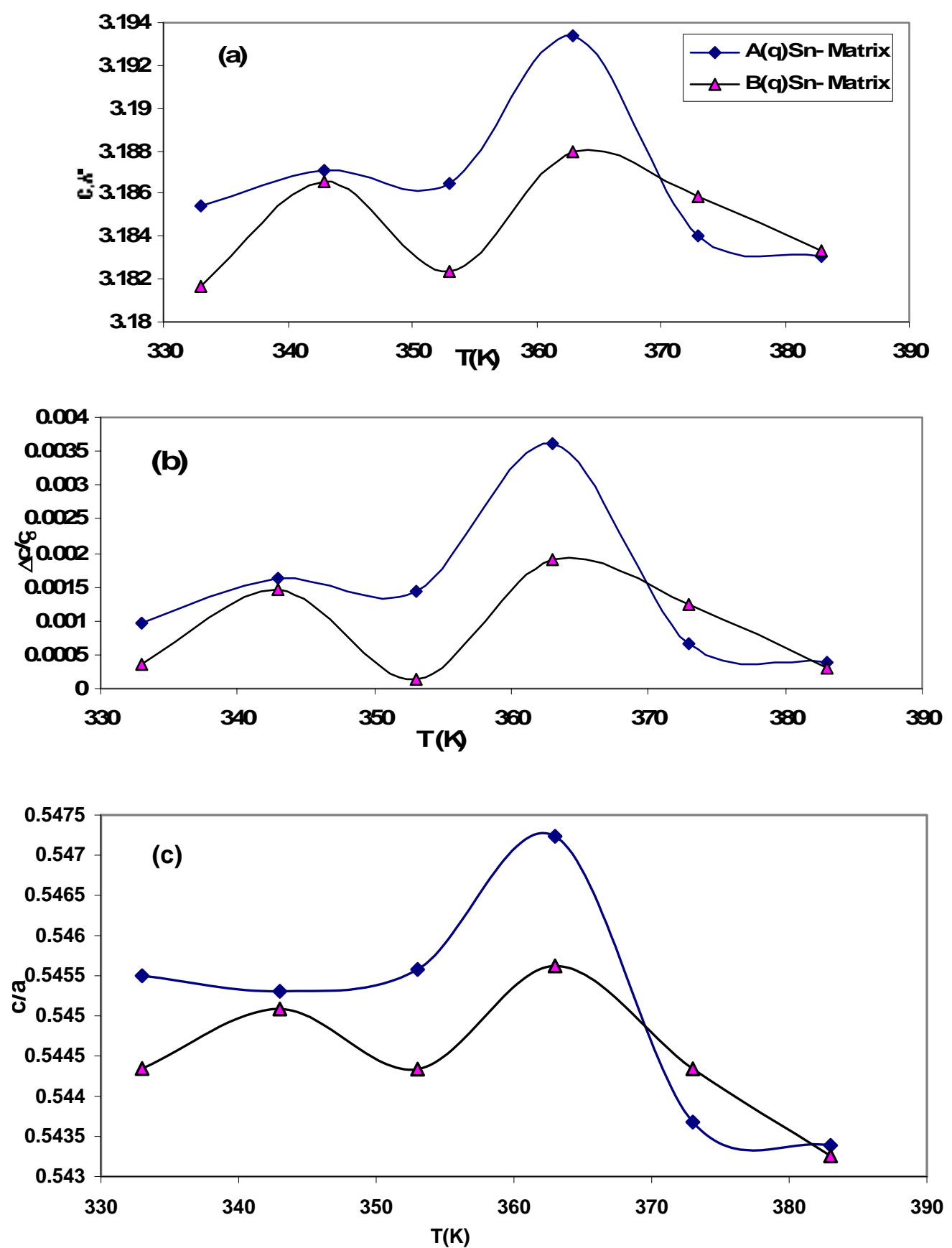

Fig. (9a,b): The effect of temperatures on the lattice parameter $\mathrm{c}$ and the residual lattice strain $\frac{\Delta c}{c}$ for both alloys. 
In normal solid solution although the solute atoms at certain state distribute randomly, they occupy definite positions at lattice sites, i.e, their disordered arrangement is changed to ordered one through ordering process. At high temperature the atoms of the two phases occupy indifinite points in the lattice, i.e, they are arranged statistically. Ordering takes place on decreasing temperature, but is usally incomplete.

Such compounds are intermidiate between chemical compounds and solid soliutions . In $\mathrm{Sn} \mathrm{Sb}$ or $\mathrm{Sn} \mathrm{Bi}$ alloys, intermediate compoundes such as $\mathrm{SnBi}$ and $\mathrm{SnSb}$ were detected in quenched sample besides, free $\mathrm{Bi}$ and $\mathrm{Sb}$ exist in their binary and ternary systems [22].

In the solid solution, the solvent retains its original; crystal lattice, but there exists a relative strengthening, as clear from the higher strength of the quenched samples than the slowly cooled ones, Fig. $(2,3)$. The solute atoms distort the solvent lattice and change the average size of its elementary cell.

The increased strengthening which results due to decrease in lattice constant is stronger than that due to an increase in lattice constant. Accordingly, the strengthening due to $\mathrm{Bi}$ of radius $(156 \mathrm{~nm})$ in $\mathrm{Sn}(151 \mathrm{~nm})$, will be smaller than that due to $\mathrm{Sb}(.145 \mathrm{~nm})$ in $\mathrm{Sn}$, which has small radius than $\mathrm{Sn}$. This is clear in Fig. $(2,3)$ and $(5)$, where the ternary samples are harder than the Sb-free samples.

In view of the phase diagram, Fig.(1), heating both alloys at $443 \mathrm{~K}$ then quenched to room temperature in cold water yield specimens of solid solution composition with very fine structure, high dislocation density and quite complex grain boundary structure [15]. This consists with the high flow stress and less ductility observed at low temperature, Fig. 2(c) for the quenched binary samples, as compared with Fig. 2(a), for the slowly cooled binary samples. The flow stress decreases and the ductility increases at high deformation temperatures. In the solid solution which maintains excess quenched in vacancies in supersaturated state, there may exist some of the following interactions which may contribute to hardening such as: short and long ranges, chemical, elastic or electrostatic and Snoek ordering. Heating the quenched samples at temperature below the line of solubility gives rise to controlled precipitation of the second phase with a high level of ductility and fine structure [23].

The characteristics of the stress-strain curves behaviour in Fig.(2) which is the same for both the slowly cooled an /or quenched samples of $\mathrm{Sn}-12 \mathrm{wt} \% \mathrm{Bi}$ and $\mathrm{Sn}-12 \mathrm{wt} \% \mathrm{Bi}-1 \mathrm{wt} \% \mathrm{Sb}$ were found to depend on the formation and/or dissolution of $\mathrm{Bi}$ and $\mathrm{Sb}$ phases in the matrix $\mathrm{Sn}$ at different temperatures . 
All the tested samples showed two relaxation stages in the temperature ranges, from $333 \mathrm{~K}$ to $353 \mathrm{~K}$ for the first stage and the second stage from $363 \mathrm{~K}$ to $413 \mathrm{~K}$. These stages are separated by a hardening peak starting from $353 \mathrm{~K}$ up to the peak at $363 \mathrm{~K}$. These stages were reported before [11] for Sn-5wt. \%Bi alloy extending in the temperature ranges: $308-328 \mathrm{~K}, 338-361 \mathrm{~K}$ and $361-388$, respectively.

According to these results [11], and the phase diagram given in Fig.(1), it can be accepted that the $\mathrm{Sn}-12 \mathrm{wt} \%$ Bi alloy shows that the $[\alpha-$ phase (rich in $\mathrm{Bi})+\mathrm{Sn}$ matrix], [ $\alpha$ - phase (rich in $\mathrm{Bi})+\beta$-phase (rich in $\mathrm{Sn}$ )] and [solid solution of single phase ( $\beta$-phase), formed by dissolution of the $\alpha$ - phase and converting it to solid solution], as structures corresponding to the three temperature stages in the present work.

As the behaviour of both $\mathrm{Bi}$ and $\mathrm{Sb}$ atoms is the same for migration towards the Sn grain cores, the maxima or the minima of the measured parameters for the binary alloy, Figs. $(3,4)$, are intensified with the $\mathrm{Sb}$ containing samples under the same conditions.

The analysis of X-ray diffraction patterns obtained for samples from the binary and tertiary alloys heat treated in the temperature range $333-383 \mathrm{~K}$ then quenched to room temperature, revealed the presence of the matrix $\mathrm{Sn}$. The integral intensity $(\mathrm{I})$, and the half line width at maximum intensity $(\Delta 2 \theta)$ The temperature dependence of the lattice constants (a and c), the ratios (c/a), besides the residual lattice strains $\left(\frac{\Delta a}{a}, \frac{\Delta c}{c}\right)$ obtained for the matrix Sn, are given in Figs.(7- 9), respectively.

Below $333 \mathrm{~K}$, as was reported $\alpha$-phase (Bi -rich phase) and $\beta$-phase ( $\mathrm{Sn}$-rich phase) coarsen and the solubility of $\mathrm{Bi}$ in $\mathrm{Sn}$ increases with increasing the testing temperature until the transition temperature observed at $(333 \mathrm{~K})$ after which the $\beta$-phase dissolves completely [10]. This increase in solubility, leading to the $\alpha$-phase (rich in $\mathrm{Bi}$ ) + Sn-phase structure, may cause the decrease in the lattice parameters, a and $\mathrm{c}$, and their residual strains, $\frac{\Delta a}{a}, \frac{\Delta c}{c}$, to reach the values of these parameters given at $333 \mathrm{~K}$ in figs.8, 9, and contribute to the softening observed in the hardening parameters of fig.3, and the increase in the softening parameters of fig. 4 at higher temperatures.

This softening may be attributed to the redistribution and coarsening of $\beta$ phase rich particles, associated with grains of increased diameters [10]. 
It is observed that the tertiary alloy containing Sb behaves the same as $\mathrm{Bi}$ at different temperatures but with different values since both $\mathrm{Bi}$ and $\mathrm{Sb}$ have the same surface activity nature [13].

In the temperature range $353 \mathrm{~K}-363 \mathrm{~K}$, the parameters, a and $\frac{\Delta a}{a}$, decreased while the parameters related to the parameter, $\mathrm{c}$, increased. This shows that the crystal lattice shrunk in the basal plane and expanded in the c-direction with the formation of, $\alpha$ - phase (rich in $\mathrm{Bi})+\beta$-phase ( $\mathrm{Sn}$-rich phase).

A redistribution process of $\mathrm{Bi}$ and /or $\mathrm{Sb}$ solute atoms in the matrix $\mathrm{Sn}$ may take place depending on the existing medium and the external factors such as temperature and stress, which may causes some of the solute atoms to be less activated at the quenching temperature and the low temperature, $363 \mathrm{~K}$, to reach their expected positions at the grain cores as states the surface activity theory [13]. Therefore, it is probable that some solute atoms will disperse near the $\mathrm{Sn}$ grain boundary regions and pin mobile dislocations leading to this increased strengthening. It is clear that the strengthening due to $\mathrm{Sb}$ is still higher than that of $\mathrm{Bi}$ as observed in fig.3.

Above $363 \mathrm{~K}$, the parameters, a and $\frac{\Delta a}{a}$, still increase, while the parameters related to the parameter, $\mathrm{c}$, still decrease, with the formation of the single phase, $\beta$-phase ( $\mathrm{Sn}$-rich phase), due to the dissolution of $\beta$-rich particles and the homogenization in the alloy. This state leads to the rapid softening of the alloy samples with increasing temperature, Figs. $(3,4)$.

The coarsening of the grains causes increased values of resilience, $U_{R}$, as large grain area needs higher amounts of energy than that in smallest one, Fig.(3-f). On the other hand, the decrease of toughness, $u_{\mathrm{f}}$, with the increase of grain diameter is expected as the large grain has a boundary of less dislocation density, i.e., of lower binding energy.Fig.(3-g).

Table (1) summarizes the present work in terms of both the softening coefficient $\alpha$ and the intrinsic hardness $\mathrm{H}_{\mathrm{o}}$ at $0 \mathrm{~K}$, which vary according to the different alloy composition and heat treatment regime which is the same for both alloys. It is well known that the defects in the quenched and tertiary samples are more than those in the slowly cooled binary samples, [5, 23]. This consist with the lower values of $\mathrm{H}_{\mathrm{o}}$ and higher values of $\alpha$ for the slowly cooled sample in the low temperature range. It is observed that the values of $\alpha$ are higher for the tertiary samples. This may due to the presence of the $\mathrm{Sb}$, having the same surface activity behaviour of $\mathrm{Bi}$ in $\mathrm{Sn}$ [13], which enhances softening in the tertiary samples, Fig. $(3,4)$. 
In the high temperature range, $\alpha$ showed a constant value of .02 , which is higher than the values obtained in the low temperature range. This may be due to the dissolution of the $\beta$-rich particles and the homogenization in the alloy - in which the parameters a, $\frac{\Delta a}{a}$ increased, fig.8, and c,$\frac{\Delta c}{c}$ decreased, Fig.(9) leading to the rapid softening observed in the tested alloys, Figs. $(2-5 a, 8,9)$.

The different values of $\mathrm{H}_{\mathrm{o}}$ in Table (1), suggest that the history of heat treatment regime applied to both alloys which have different components, i.e., of initial different states, should lead to final different states at $0 \mathrm{~K}$, if reached, with different characteristics rather than common single state as wrongly may be expected at $0 \mathrm{~K}$.

\section{Conclusion:}

Stress-strain test for Sn-12wt. \%Bi and the Sn-12wt. \%Bi-1wt. \%Sb samples was carried out in the temperature range from (333-413) K, and x-ray diffraction patterns were obtained at room temperature for both alloys treated in the range (333-383) $\mathrm{K}$.

Two relaxation stages at, (333-353) $\mathrm{k}$ and (363-413) $\mathrm{k}$ were observed separated by a hardening peak, (353-363) K.

The temperature dependence of the hardening parameters for the tested alloys decreased to minima at $353 \mathrm{~K}$ followed by maxima at $363 \mathrm{~K}$ then showed continuous decrease up to $413 \mathrm{~K}$. The softening parameters showed a reversed behaviour. Increasing temperature and $\mathrm{Sb}$ addition lead to softening.

The parameters measured at $333 \mathrm{~K}$ are due to reported softening attributed to redistribution and coarsening of $\beta$-rich particles. Above $333 \mathrm{~K}$ the softening was due to the dissolution of Bi-rich particles in the $\alpha$-phase and the homogenization of the alloy.

In the temperature range (353-363) $\mathrm{K}$ the parameters a, $\frac{\Delta a}{a}$ decreased and c, $\frac{\Delta c}{c}$ increased. Above $363 \mathrm{~K}$ a, $\frac{\Delta a}{a}$ increased and $\mathrm{c}, \frac{\Delta c}{c}$ decreased.

In the low temperature range, $\alpha$ increased with $\mathrm{Sb}$ addition and its values were higher for the slowly cooled samples than the quenched samples. $\mathrm{H}_{\mathrm{o}}$ was higher for the tertiary and quenched samples all over the tested temperature. 
In the high temperature range, $\alpha$ showed a constant value of .02, and Ho showed different values at $0 \mathrm{~K}$ due to the different initial states of the tested samples. This ensures the effect of temperature and $\mathrm{Sb}$ addition on the final state of the tested material at $0 \mathrm{~K}$, if reached, which can be misunderstood at $0 \mathrm{~K}$.

\section{References:}

1. Y-S Kim, K-S Kim, C-W Hwang, K Suganuma, Journal of Alloys and Compounds, 352, 237 (2003).

2. B.T.K. Barry, C.J. Thwattes, Tin and Its Alloys and Compounds, Wiley, New York, pp.280 (chapters 3-4) (1983).

3. J.W. Yoon, C.B. Lee, S.B. Jung, Mater. Sci. Technol. 19, 1101 (2003).

4. S. K. Kang, P. A. Lauro, D.-Y. Shih, D. W. Henderson, K. J. Puttlitz, IBM J. RES. \& DEV. 49, 4, (2005).

5. F. Abd El-Salam, A.M. Abd El-Khalek, R.H. Nada, A. Fawzy. Mat. Characterization 59, 9 (2008).

6. K.suganuma, Curr.Opin. Solid State Mater. Sci. 5, 55 (2001).

7. M. Dragan, V. JanV, M. Du`skoi'c, K. Ale`s, Z. Dragana, Z. `Zivan`, J. Alloys and Compounds 438, 150 (2007).

8. G.S. Al-Ganainy, M.R. Nagy, B.A. Khalifa, R. Afifi, Phys. Stat. Sol. (a) 158, 463(1996).

9. G. Saad, F. Abd El-Salam, M.T. Mustafa, Surf. Technol. 23, 73 (1984).

10. C.C. Lee, R.W. Chuang, D.W. Kim, Mater. Sci. Eng. A 374, 280 (2004).

11. B.A.Khalifa, M.R.Nagy, G.S.Al-Ganainy and R.Afify. Egypt. J. Solids, 29, 1 (2006).

12. A.F.Abd El-Rehim, J. Alloys and Coompounds, 440, 127 (2007).

13. V. K. Semenchenko, "Surface Phenomena in Metals and Alloys", Pergamon, London (1961).

14. M.Hansen, K.Anderko, constitution of binary alloys. New York; Mc Grew -Hill Book Co.Inc.; p.337 (1958).

15. F. Abd El-Salam, A.M. Abd El-Khalek, R.H. Nada, Physica B 292, 71 (2000).

16. M.M. Mostafa, Czech J. of Physics. 51, 3 (2001).

17. R.H. Nada, Physica B 349, 166 (2004).

18. M. Kerr, N. Chawla, Acta Materialia 52, 4527 (2004).

19. M. A. E. Shereen, M.Sc. Thesis, Fac. Education, Ain Shams Univ. Egypt, a)p.43, -b)p.44 (2003).

20. H.Y. Zahran, M.Sc. Thesis, Physics Dept., Fac. Education, Ain Shams Univ. p.32 (2006).

21. F. Abd El-Salam, A.M. Abd El-Khalek, R.H. Nada, L.A. Wahab, H.Y. Zahran, Mater. Sci. Eng. A 527, 1223 (2010). 
22. F. Abd El-Salam, R.H. Nada, A.M. Abd El-Khalek, M.R.Nagy, R.Abd El-Haseeb, Mat. Sci. Eng. A527, 2962 (2010).

23. F. Abd El-Salam, M.M. Mostafa, L.A. Wahab, M.T. Mostafa, M. A. E. Shereen. Mat Sci Eng A 478, 71 (2008). 